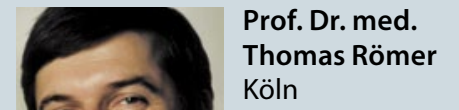

Prof. Dr. med. Köln

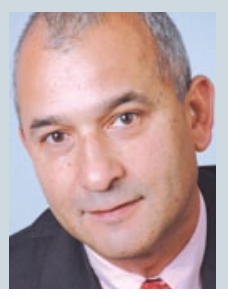

Prof. Dr. med.

Tino F. Schwarz

Würzburg

\section{Roboter braucht länger für die Myomektomie}

\section{Ob mit oder ohne Roboterhilfe - die Ergebnisse der laparoskopischen Myomektomie unterscheiden sich kaum. Doch der Roboter war in einer Studie deutlich langsamer als die Kollegen aus Fleisch und Blut. Vermutlich lag das am Nahtmaterial.}

G ynäkologen haben die Ergebnisse nach laparoskopischer Myomektomie in einer retrospektiven Kohortenstudie untersucht. 115 Eingriffe waren in üblicher minimalinvasiver Technik erfolgt, an 176 Operationen war ein Operationsroboter vom Da-Vinci-Typ beteiligt gewesen.

Beide Gruppen waren hinsichtlich der Altersverteilung, des Body-Mass-Index und der Leiomyommerkmale vergleichbar. Auch die operativen Ergebnisse unterschieden sich nur wenig. Die Reoperationsrate lag bei $0,9 \%$ nach Standardtechnik und bei $0,6 \%$ nach Roboterassistenz. Postoperative Komplikationen traten bei $14,5 \%$ respektive $8,6 \%$ der operierten Frauen auf. Keiner dieser Unterschiede war signifikant.

Statistisch bedeutsame, aber klinisch wohl unerhebliche Differenzen gab es beim Blutverlust, der im Mittel 85,3 ml (Standard) bzw. 112,2 ml (Roboter) betrug $(\mathrm{p}<0,04)$. Die Operationszeit allerdings unterschied sich deutlich, und zwar zuungunsten der Roboterhilfe. Während herkömmliche laparoskopische Eingriffe im Mittel nach 118,3 Minuten beendet waren, dauerten Roboterunterstützte Myomektomien 195,1 Minuten.

Eine etwaige Lernkurve, die sich beim Roboter-gestützten Vorgehen bemerkbar machen könnte, war dabei berücksichtigt. Sie kann die längere Operationszeit daher nicht erklären. Diese ist nach Ansicht Autoren vielmehr auf die eingesetzte Nahttechnik zurückzufüh- ren. Nach Eingriffen mit Roboterhilfe wurden die Gewebelagen in 95\% der Fälle wie nach einer offenen Operation verschlossen. Nach der laparoskopischen Standardoperation wurde hingegen bei $70 \%$ Nahtmaterial mit Widerhaken verwendet. Es macht das Knüpfen von Knoten überflüssig und beschleunigt den Wundverschluss.

Die operativen Ergebnisse der Roboter-gestützten Myomektomien gleichen diesen Ergebnissen zufolge jenen, die mit laparoskopischen Standardeingriffen $\mathrm{zu}$ erzielen sind. Unterschiede im Blutverlust sind zu vernachlässigen, längere Operationszeiten vermutlich der Nahttechnik zuzuschreiben. Voraussetzung ist aber immer, dass die Operationen von erfahrenen Teams vorgenommen werden.

Dr. Robert Bublak

Gargiulo AR et al. Robot-Assisted Laparoscopic Myomectomy Compared With Standard Laparoscopic Myomectomy. Obstet Gynecol 2012; 120: 284-91

Kommentar: Die Arbeit bearbeitet die interessante Fragestellung, ob die roboterassistierte Chirurgie bei Routineeingriffen, wie der Myomektomie, Vorteile bringt. Zwei wesentliche Unterschiede finden sich zwischen beiden Gruppen. Der erhöhte Blutverlust bei der laparoskopischen Myomektomie mit dem Roboter ist zwar statistisch signifikant, aber klinisch bei einer Differenz von etwa $25 \mathrm{ml}$ kaum relevant. Allerdings findet sich in der Gruppe der Patientinnen mit der roboterassistierten Myomektomie

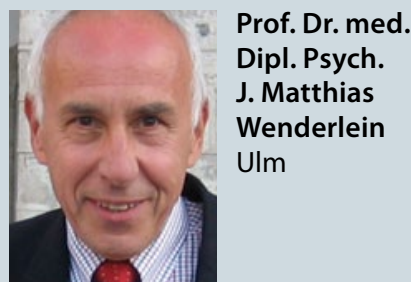

eine Bluttransfusionsrate von 5,7\% (10 Frauen). Diese ist signifikant höher als in der anderen Gruppe und für laparoskopische Myomenukleationen doch als relativ hoch zu bewerten. Auch wenn dies mit präoperativ chronischen Anämien begründet wird, ist dies jedoch als nachteilig zu bewerten. Die Operationszeit mit der Roboterhilfe war signifikant länger. Da dies in ähnlichen Studien nicht der Fall war, wird dies mit den unterschiedlichen Nahttechniken begründet. Insofern ist diese vergleichende Studie insgesamt nur eingeschränkt verwertbar, da besonders die Nahttechnik den größten Zeitfaktor bei einer laparoskopischen Myomenukleation darstellt. Auch der etwas höhere Blutverlust lässt sich möglicherweise dann durch die unterschiedlichen Nahttechniken erklären. Ein weiterer Faktor, der in der Arbeit nicht berücksichtig wird, sind die Kosten für die jeweilige Technik, die bei den Roboter-Operationen um ein vielfaches höher liegt. Hinzu kommt, dass die mit Roboter operierten Patientinnen der Studie häufig hospitalisiert werden mussten bzw. einen längeren stationären Aufenthalt hatten.

Die Studie lässt zumindest keinerlei Vorteile einer roboterassistierten laparoskopischen Myomenukleation gegenüber der herkömmlichen Technik erkennen. Wenn man den Kostenfaktor noch mit heranzieht, dürften die Nachteile derzeit überwiegen. Insofern bedarf es weiterer prospektiver Studien, um wirklich einen klaren Vorteil der roboterassistierten laparoskopischen Myomektomie herauszustellen, welche die deutlich höheren Kosten rechtfertigen würden. Insofern sollte diese Methode derzeit nur im Rahmen von Studien angewendet werden. Ansonsten muss die Patientin aufgrund der Datenlage über längere Operationszeiten und höheren Blutverlust aufgeklärt werden.

Prof. Dr. med. Thomas Römer 